\title{
The roles of stiffness and excluded volume in DNA denaturation
}

\author{
Enrico Carlon ${ }^{(1)}$, Enzo Orlandini ${ }^{(1)}$ and Attilio L. Stella ${ }^{(1,2)}$ \\ (1) INFM, Dipartimento di Fisica, Università di Padova, I-35131 Padova, Italy \\ (2) Sezione INFN, Università di Padova, I-35131 Padova, Italy
}

(October 31, 2018)

\begin{abstract}
The nature and the universal properties of DNA thermal denaturation are investigated by Monte Carlo simulations. For suitable lattice models we determine the exponent $c$ describing the decay of the probability distribution of denaturated loops of length $l, P \sim l^{-c}$. If excluded volume effects are fully taken into account, $c=2.10(4)$ is consistent with a first order transition. The stiffness of the double stranded chain has the effect of sharpening the transition, if it is continuous, but not of changing its order and the value of the exponent $c$, which is also robust with respect to inclusion of specific base-pair sequence heterogeneities.
\end{abstract}

PACS numbers: 87.14.Gg, 05.70.Jk, 05.70.Fh, 87.15.Aa

The melting of a DNA molecule from a double stranded phase to a denaturated state where the two strands are unbound has been the subject of various studies in the past [1 -8]. Experiments done with UV absorption by diluted DNA solutions show that denaturation occurs through a series of jumps in the absorbance spectrum as a function of temperature [9]. The jumps are interpreted as sudden denaturations of large double stranded portions of the inhomogeneous chain and suggest a first order character for the transition. The existing models, even if believed to capture the relevant features of the system, introduce drastic simplifications of the complex structure of the DNA molecule in favor of analytical tractability. Within these models, two different mechanisms responsible for a first order transition were recently proposed. For the Peyrard-Bishop (PB) model [3], it has been argued that the stronger stiffness of double stranded compared to single stranded DNA, may lead to a first order denaturation [5]. It has also been proposed that this stiffness difference, in combination with base sequence heterogeneity, should be responsible for the observed jumps in absorption 任. Other studies using the Poland-Sheraga (PS) [1] model led to the claim that excluded volume effects, even in the absence of stiffness, induce first order melting [7].

In this Letter we investigate further these issues within models representing as realistically as possible the relevant properties of DNA. Within such models all mechanisms mentioned above can operate and thus be tested simultaneously without resorting to uncontrollable approximations. Near the transition DNA can be regarded as an alternating sequence of double helix segments along which base pairs are bound, and of denaturated loops, where the two strands are detached. By a scaling analysis of the cumulative probability distribution function of denaturated loop lengths we show clearly that excluded volume effects drive the transition first order, in the limit of infinitely long chains. We also find that double helix stiffness alone does not modify such asymptotic result in a range of values chosen consistently with those expected for real DNA. However, in the presence of strong stiffness, and for a model with second order denaturation in the infinite chain limit, the critical region becomes very narrow and, due to a slow crossover, thermodynamic quantities like the specific heat may behave consistently with first order even for long finite chains.

We model the DNA strands by two self-avoiding walks (SAW) of length $N$ on cubic lattice, identified by the vectors $\vec{r}_{1}(i)$ and $\vec{r}_{2}(i)(0 \leq i \leq N)$, joined at a common origin $\left(\vec{r}_{1}(0)=\vec{r}_{2}(0)\right)$ and with free endpoints. A gain of energy $\varepsilon$ (=1 here) is associated to a bond between the two strands, which occurs in the model when two monomers with the same $i$ overlap $\left(\vec{r}_{1}(i)=\vec{r}_{2}(i)\right)$. The binding energy is taken to be the same all along the chain, i.e., to start with, we neglect the heterogeneity of base pair interactions of specific sequences. At sufficiently low $\mathrm{T}$ the most probable configurations are fully bound, i.e. $\vec{r}_{1}(i)=\vec{r}_{2}(i)$ for all $i$. Upon increasing the temperature the two strands are expected to unbind at some $T=T_{c}$. The transition is driven by the formation of denaturated loops, whose length can be measured by the number of unbound monomers, $l$, of the corresponding strand segments.

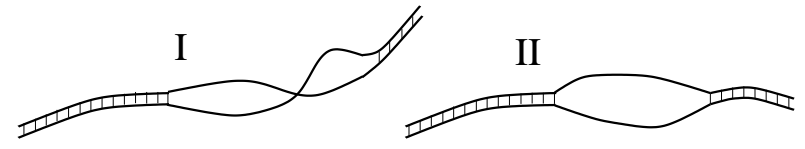

FIG. 1. Schematic representations of denaturated loops in model I and II.

In order to investigate in detail excluded volume and stiffness effects we consider two different models, which we refer to as model I and II. While in both models bounded segments are self-avoiding and cannot overlap any other part of the chain, in I the excluded volume condition is partly relaxed (see Fig. 1): the two strands forming a denaturated loop are self-, but not mutually avoiding and overlap at non-complementary sites (i.e. $\vec{r}_{1}(j)=\vec{r}_{2}(k)$ with $\left.j \neq k\right)$ are allowed. Such overlaps do not contribute to the energy. The stiffness of the double stranded chain is incorporated in a second parameter 
$\varepsilon_{b}$, which is the energy gain if two consecutive segments in the bound chain are aligned (i.e. when $\vec{r}_{1}(i)=\vec{r}_{2}(i)$ for $i=j-1, j, j+1$ and $\left.\vec{r}_{1}(j+1)-\vec{r}_{1}(j)=\vec{r}_{1}(j)-\vec{r}_{1}(j-1)\right)$. Of course, both models neglect the precise helix structure. Model II, having excluded volume effects fully built in, is a more realistic representation of DNA. A recent study, in absence of stiffness [6], claimed for it some evidence of first order transition.

For models I and II we sampled by a multiple Markov chain Monte Carlo (MC) [10 method the loop length probability distribution function (pdf) at several different temperatures. In all cases the pdf has an exponential decay at low $\mathrm{T}\left(P(l, N) \sim \exp \left(-l / l_{0}\right)\right)$ where bound segments are instead broadly distributed in length. From a given $T_{c}$ upwards one observes instead a scaling form $P(l, N) \sim l^{-c} f(l / N)$, where the exponent $c$ seems to be approximately independent of $T$ and $f$ is a scaling function. The length of bound double segments is narrow distributed in this case. The general picture emerging from our numerical results has an immediate thermodynamical interpretation. Quantities usually analyzed at polymer conformational transitions are the energy per monomer or the specific heat, which for a chain of length $N$ and $T \sim T_{c}$ scales as 11]:

$$
\mathcal{C}(N, T) \sim N^{2 \phi-1} h\left[\left(T-T_{c}\right) N^{\phi}\right]
$$

where $h$ is a scaling function and $\phi$ is the crossover exponent. For large $N$ one has $\mathcal{C}_{\max }(N)=\max _{T} \mathcal{C}(N, T) \sim$ $N^{2 \phi-1}$, from which $\phi$ can be deduced. The density of binding contacts along the strands, proportional to the energy, should scale as $N^{\phi-1}$ at $T=T_{c}$. Clearly $\phi \leq 1$, and only $\phi=1$ implies a first order discontinuity of the density. Given the scaling form of $P(l, N)$ and the fact that bound segment lengths are finite on average at $T_{c}$, the same density should also scale as the reciprocal of the average loop length $\langle l\rangle=\sum_{l} l P(l, N)$. Now, for $1 \leq c<2,\langle l\rangle \sim N^{2-c}$, so that $\phi=c-1<1$, and the transition is second order. If instead $c>2,\langle l\rangle$ and the energy density remain finite at $T_{c}$ for $N \rightarrow \infty$ and the transition is first order $(\phi=1)$. Analyzing $P$ offers both fundamental and practical advantages over the use of Eq. (11). Indeed, due to finite size corrections to scaling, a reliable estimate of $\phi$ requires good determinations of $\mathcal{C}$ around $T_{c}$ for sufficiently long chains [12]. On the contrary, the scaling behavior of $P$ sets in already for relatively short chains $(N \approx 100)$, which allow us to estimate $c$ reliably. The robustness of the estimate with respect to the variation of chain lengths assures that results are little affected by finite size corrections.

We consider first $\varepsilon_{b}=0$. Figure 2 shows log-log plots of $P$ as a function of $l$ for $N=50,100$ and 180 for model I, at $T=0.85 \approx T_{c}$ (we estimate $T_{c}=0.85(2)$ ). After an initial transient the data follow nicely a straight line in the plots, except when $l \approx N$, where of course $P$ drops. It is interesting to note that the power-law regime sets in already for relatively short loops. A linear fit of the data for $N=180$ gives $c=1.73(4)$, indicating a continuous denaturation for model I. By fitting
$\mathcal{C}_{\max }$ as $\mathcal{C}_{\max }(N)=A N^{2 \phi-1}$ (inset of Fig. 2), we obtain $\phi=0.77(2)$. Thus again $\phi<1$ indicates a continuous transition; moreover, the above proposed relation $\phi=c-1$ is well satisfied.

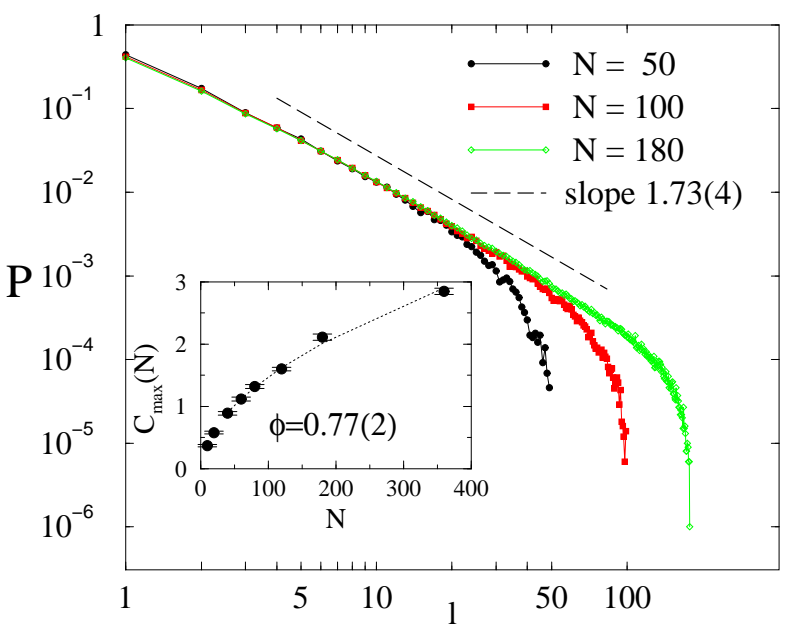

FIG. 2. Plot of $P$ vs. $l$ on a log-log scale for model I at $T=T_{c}$ and various chain lengths $N$. Inset: $\mathcal{C}_{\max }$ as a function of $N$ and relative fit $\sim N^{2 \phi-1}$ (see Eq. (1)).

Within a PS model description each loop would be assumed to be totally uncorrelated with the rest of the chain, which at $T \geq T_{c}$ would just provide a grandcanonical critical reservoir of strand elements to form the loop. The value of $c$ within this scheme can be calculated easily. The critical grand-canonical pdf of a SAW of length $l$ with fixed end-to-end distance $R_{e}$, in three dimensions, is [11]:

$$
p\left(l, R_{e}\right) \sim l^{\gamma-3 \nu-1} g\left(R_{e} l^{-\nu}\right),
$$

where $g$ is a scaling function, $\gamma$ is the entropic, and $\nu$ is the metric exponent. A loop in model I is made of two strands with common end-points and allowed to intersect each other. Thus the probability of a loop of length $l$ (total perimeter $2 l$ ) is:

$$
P(l) \sim \int d^{3} R_{e} p^{2}\left(l, R_{e}\right) \sim l^{2 \gamma-3 \nu-2}
$$

from which follows $c^{(\mathrm{IL})}=2+3 \nu-2 \gamma$, where IL stands for isolated loop. Using the appropriate SAW exponents, i.e. $\gamma \approx 1.158, \nu \approx 0.588$ [11], we find $c^{(\mathrm{IL})} \approx 1.448$ [13]. This result allows us to quantify the variation of the exponent $c$ due to excluded volume interactions between loops and segments which are taken into account in the MC simulations, namely $\Delta c=c-c^{(\mathrm{IL})} \approx 0.3$.

We now turn to model II. Fig. 3 shows the loop probability distribution at $T \approx T_{c}\left(T_{c}=0.76(2)\right)$ for $N=50$, 100, 150 and 200. From a linear fit of the data we obtain $c=2.10(4)>2$. Excluded volume effects appear to be responsible for the discontinuous nature of denaturation, 
in agreement with a recent extension of the PS model in which the interaction between loops and segments was included in an approximate way [7]. By relying on field theoretical results for polymeric networks [14], the authors of Ref. [7] estimated analytically the loop length pdf exponent to be $c \approx 2.12$, which is remarkably close to our numerical determination, suggesting that the sort of perturbative treatment of Ref. [7] catches the essential contribution to the correlations among different loops and segments. The inset of Fig. 3 shows a plot of $P$ for $T=1.0$, i.e. well above $T_{c}$, and $N=150$; the data still show a power-law behavior with an exponent $c=2.09(5)$, i.e. consistent with the value found at $T_{c}$.

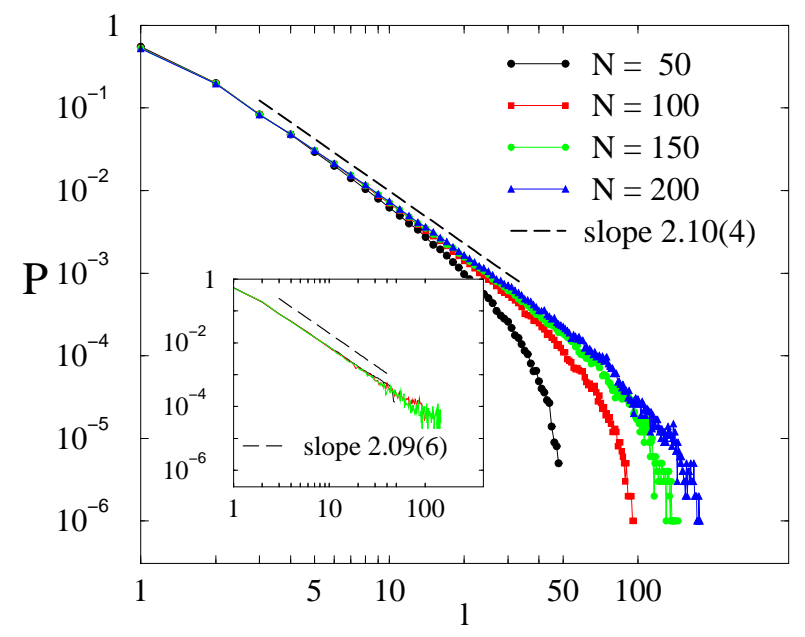

FIG. 3. Log-log plots of $P$ vs. $l$ for model II at $T=T_{c}$ and for various $N$. Inset: Same plots for $T=1.3 T_{c}$.

TABLE I. Summary of exponents found for the two models.

\begin{tabular}{c|ccc}
\hline \hline Model & $c^{(\mathrm{IL})}$ & $c\left(\varepsilon_{b}=0\right)$ & $c\left(\varepsilon_{b}=5\right)$ \\
\hline I & 1.448 & $1.73(4)$ & $1.70(6)$ \\
II & 1.762 & $2.10(4)$ & $2.06(6)$ \\
\hline \hline
\end{tabular}

Table summarizes the results obtained for the two models. In three dimensions the exponent of an isolated loop is $c^{(\mathrm{IL})} \approx 1.762[11$. For both model I and II the excluded volume interactions are responsible for a roughly identical increase of $c$ with respect to its "bare", isolated loop value $c^{(\mathrm{IL})}$. For model I this effect is not strong enough to cause a first order transition.

Next we ask whether a sufficiently strong $\varepsilon_{b}>0$ may induce first order denaturation. Recently such a possibility has been discussed in the context of the PB model [4. [. This model, like the PS one, takes into account excluded volume effects very inadequately, since the two strands, while prevented from overlapping each other, are in fact not embedded in ordinary three dimensional space. Only a sort of longitudinal coordinate along the DNA backbone and the distance between strands enter the description. Within this context it was predicted that the stiffness of bound segments sharpens a continuous transition, making it look like first order for practical purposes $[4$, or even drives it strictly first order [5].

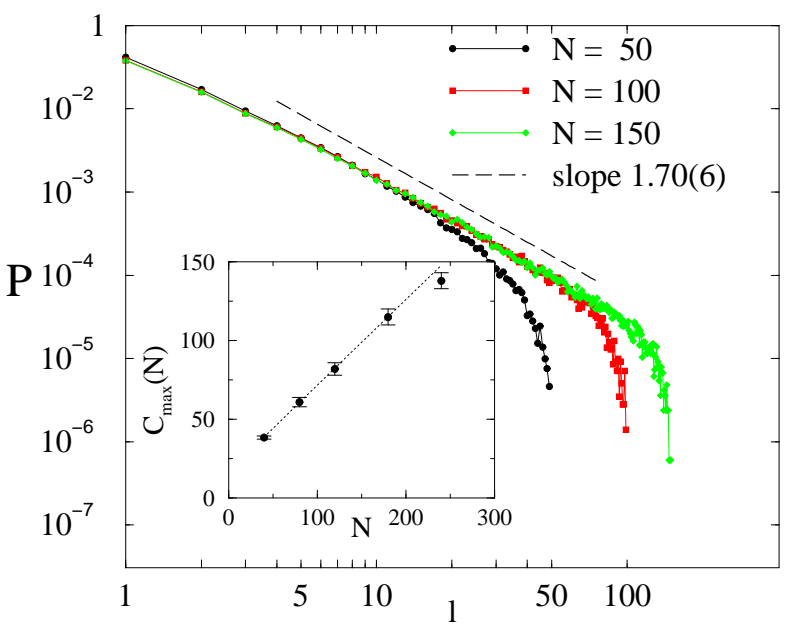

FIG. 4. Log-log plot of $P$ vs. $l$ for model I with $\varepsilon_{b}=5$. Inset: scaling of $\mathcal{C}_{\max }$ as a function of $N$.

We focus first on model I, which has a continuous transition for $\varepsilon_{b}=0$. Figure 1 shows a plot of $\ln P$ vs. $\ln l$ for $\varepsilon_{b}=5$. From a linear fit we obtain $c=1.70(6)$, in agreement with the value determined for $\varepsilon_{b}=0$. This indicates that a value of stiffness $\varepsilon_{b}=5$ does not change the asymptotic character of the transition, which remains second order and in the same universality class as for $\varepsilon_{b}=0$. The inset of Fig. 目 shows a plot of $\mathcal{C}_{\max }$ as a function of $N$. This quantity scales linearly up to $N \approx 200$, and deviates from linearity only for the longest lengths investigated. A linear scaling would imply $\phi=1$, i.e. a first order transition. Apparently there is a very slow crossover in the specific heat, i.e. the deviation from $\mathcal{C}_{\max }(N) \sim N$ can be observed only for very long chains. Indeed, for $\varepsilon_{b}=10$, which can also be a realistic value for DNA, the crossover must occur at $N$ values which are not accessible to our simulation. Notice that, on the contrary, already for rather short chains $(N \lesssim 100)$ the behavior of $P$ indicates clearly that $c \sim 1.7<2$. So, while the investigation of $\mathcal{C}$ in the presence of realistic stiffness would reveal the true asymptotic nature of the transition only for extremely long chains, $c$ seems to be very little affected by crossover. This discrepancy is due to the different way in which finite size effects and temperature uncertainties affect the scalings of $P$ and $\mathcal{C}$, as discussed above.

For model II it is not possible to detect crossover behavior in $\mathcal{C}_{\max }$, since the transition is already first or$\operatorname{der}(\phi=1)$ at $\varepsilon_{b}=0$. For $\varepsilon_{b}=5$ and $\varepsilon_{b}=10$, which, as discussed below, are realistic choices, we estimate $c=2.06(6)$ and $c=2.04(8)$, respectively. These values do not deviate, within error bars, from that obtained at $\varepsilon_{b}=0$. However, a very slight systematic decrease of $c$ with increasing $\varepsilon_{b}$ cannot be excluded. This 
decrease could be determined by a mild crossover phenomenon induced by $\varepsilon_{b}>0$. Double stranded DNA has a persistence length which is about $20-50$ times longer than that of single stranded chains 15 . According to our calculations the persistence lengths of bounded segments for $T \approx 0.8 T_{c}$ [16] are about 10 and 30 times those of the unbound segments for $\varepsilon_{b}=5$ and $\varepsilon_{b}=10$, respectively. Therefore the above parameter choices should be considered as rather realistic for DNA. A single loop attached to very stiff long segments (a typical configuration for our chains at low temperatures) should experience very little excluded volume interaction with them. However, close to denaturation more and more loops start forming and the whole chain becomes rather flexible as the bubbles carry no stiffness. Typical estimates in our simulations yield a persistence length of just $2-3$ lattice steps close to $T_{c}$, explaining why the stiffness has little effect on the critical loop pdf. For a correct physical interpretation of the models studied here one should assign to each lattice monomer about 10 base pairs (bp), thus we predict that the persistence length of the double stranded chain close to denaturation would be of the order of $20-30 \mathrm{bp}$.

We finally considered the effect of heterogeneity of the binding energies of base pairs along the chain. As an example we embodied in model II the information concerning a specific DNA sequence, resorting to a strictly microscopic interpretation of lattice monomers as single bases. We took $\varepsilon_{\mathrm{CG}} / \varepsilon_{\mathrm{AT}}=1.5$ and $\varepsilon_{b}=5$, as expected for real DNA. For two different sequences of length $N=150$ [17] we estimated $c=2.10(8)$, indicating that $c$ robustly maintains the value determined for the homogeneous version of the model.

Another important consequence of our results concerns codes 18] used to simulate melting curves of real DNA sequences. These codes contain various parameters, such as the stacking energy of neighboring base pairs, and use $c$ in the partition function for denaturated loops. The typical choice is $c \approx 1.7$ [18], which is the appropriate value for a single isolated loop, while a more consistent choice would be $c \approx 2.1$. It would be interesting to investigate the consequence of this different value of $c$ on the calculated melting curves.

Summarizing, the DNA denaturation transition can be characterized in terms of the algebraic decay of the cumulative loop length pdf. The exponent $c$ signals clearly the asymptotic nature of the transition and is in fact well defined already for relatively short chains. In all our calculations we observe an onset of power-law behavior for short loops $(l \gtrsim 5)$. This suggests that, if direct measurements could be realized, the sampling of $P$ would not need to include very long denaturated loops and rather microscopic probes could be adequate. Promising in this respect seem to be techniques based on fluorescent DNA probes (see e.g. Ref. [19]).

Excluded volume effects alone appear to be responsible for the discontinuous nature of denaturation in the infinite chain limit. Our calculations provide in fact a final verification of a conjecture advanced many decades ago by Fisher [2] and recently corroborated and made more precise by Kafri et al. [7]. The stiffness of the double stranded DNA is not responsible for the first order character, contrary to claims based on results for the $\mathrm{PB}$ model [5], but possibly only produces very mild crossover effects on $c$. In realistic conditions these effects should be barely detectable even on relatively short chains.

Financial supports by MURST-COFIN 01, INFMPAIS 01 and European Network ERBFMRXCT980183 are gratefully acknowledged.

[1] D. Poland and H. A. Sheraga, J. Chem. Phys. 45, 1456 (1966); 45, 1464 (1966).

[2] M. E. Fisher, J. Chem. Phys. 45, 1469 (1966).

[3] M. Peyrard and A. R. Bishop, Phys. Rev. Lett. 62, 2755 (1989); T. Dauxois, M. Peyrard and A. R. Bishop, Phys. Rev. E 47, R44 (1993).

[4] D. Cule and T. Hwa, Phys. Rev. Lett. 79, 2375 (1997).

[5] N. Theodorakopoulos, T. Dauxois, and M. Peyrard, Phys. Rev. Lett. 85, 6 (2000).

[6] M. S. Causo, B. Coluzzi, and P. Grassberger, Phys. Rev. E 62, 3958 (2000).

[7] Y. Kafri, D. Mukamel, and L. Peliti, Phys. Rev. Lett. 85, 4988 (2000).

[8] T. Garel, C. Monthus, and H. Orland, Europhys. Lett. 55, 132 (2001).

[9] R. M. Wartell and A. S. Benight, Phys. Rep. 85, 67 (1985).

[10] M. C. Tesi, E. J. Janse van Rensburg , E. Orlandini, and S. G. Whittington, J. Stat. Phys. 29, 2451 (1996).

[11] C. Vanderzande, Lattice Models of Polymers (Cambridge University Press, Cambridge 1998).

[12] The difficulty of a reliable determination of $\phi$ from specific heat data has been encountered in Ref. [6], where the estimate $\phi \sim 0.9$, for model II, is not fully consistent with the claimed first order nature of the transition.

[13] Notice that using the random walk exponents $\gamma=1$, $\nu=1 / 2$ in the relation $c=2+3 \nu-2 \gamma$ one obtains $c=3 / 2$, which is the correct value of $c$ for a loop where no excluded volume effects are considered. It is also interesting to point out that for a loop in model I, $c^{(\mathrm{IL})}=1.448<3 / 2$. This goes against the expectation that the incorporation of excluded volume effects always leads to an increase of the value of $c$.

[14] B. Duplantier, Phys. Rev. Lett. 57, 941 (1986).

[15] M. T. Record et al., Annu. Rev. Biochem. 50, 997 (1981).

[16] We assume a typical denaturation temperature of $T_{c} \approx$ $80^{\circ} \mathrm{C}$, therefore the room temperature, where persistence lengths are typically measured, corresponds to $\approx 0.8 T_{c}$.

[17] We used a DNA sequence from coliphage $\Phi$ X174 taken from http://www.ncbi.nlm.nih.gov/Taxonomy/.

[18] R. D. Blake et al., Bioinformatics 15, 370 (1999).

[19] G. Bonnet, O. Krichevsky, and A. Libchaber, Proc. Natl. Acad. Sci. 95, 8602 (1998). 